\title{
A comparison of two different sound intensity measurement principles
}

\author{
Jacobsen, Finn; de Bree, Hans-Elias
}

Published in:

Acoustical Society of America. Journal

Link to article, DOI:

$10.1121 / 1.1984860$

Publication date:

2005

Document Version

Publisher's PDF, also known as Version of record

Link back to DTU Orbit

Citation (APA):

Jacobsen, F., \& de Bree, H-E. (2005). A comparison of two different sound intensity measurement principles. Acoustical Society of America. Journal, 118(3), 1510-1517. https://doi.org/10.1121/1.1984860

\section{General rights}

Copyright and moral rights for the publications made accessible in the public portal are retained by the authors and/or other copyright owners and it is a condition of accessing publications that users recognise and abide by the legal requirements associated with these rights.

- Users may download and print one copy of any publication from the public portal for the purpose of private study or research.

- You may not further distribute the material or use it for any profit-making activity or commercial gain

- You may freely distribute the URL identifying the publication in the public portal

If you believe that this document breaches copyright please contact us providing details, and we will remove access to the work immediately and investigate your claim. 


\title{
A comparison of two different sound intensity measurement principles ${ }^{\text {a) }}$
}

\author{
Finn Jacobsen ${ }^{\text {b) }}$ \\ Acoustic Technology, Ørsted $\bullet D T U$, Technical University of Denmark, Building 352, Ørsteds Plads, \\ DK-2800 Kgs. Lyngby, Denmark \\ Hans-Elias de Bree ${ }^{\mathrm{c})}$ \\ Microflown Technologies BV, P.O. Box 300, 6900 AH Zevenaar, The Netherlands
}

(Received 1 November 2004; revised 1 June 2005; accepted 2 June 2005)

\begin{abstract}
The dominating method of measuring sound intensity in air is based on the combination of two pressure microphones. However, a sound intensity probe that combines an acoustic particle velocity transducer with a pressure microphone has recently become available. This paper examines, discusses, and compares the two measurement principles with particular regard to the sources of error in sound power determination. It is shown that the phase calibration of intensity probes that combine different transducers is very critical below $500 \mathrm{~Hz}$ if the measurement surface is very close to the source under test. The problem is reduced if the measurement surface is moved further away from the source. The calibration can be carried out in an anechoic room. () 2005 Acoustical Society of America. [DOI: 10.1121/1.1984860]
\end{abstract}

PACS number(s): 43.58.Fm, 43.50.Yw, 43.38.Kb [AJZ] Pages: 1510-1517

\section{INTRODUCTION}

The most successful method of measuring sound intensity in air is the "two-microphone" (or " $p-p$ ") method, which makes use of two closely spaced pressure microphones and relies on a finite-difference approximation to the sound pressure gradient. ${ }^{1}$ Both the IEC standard on instruments for the measurement of sound intensity ${ }^{2}$ and the corresponding North American ANSI standard ${ }^{3}$ deal exclusively with $p-p$ measurement systems. The alternative " $p-u$ " method, which involves combining a pressure microphone with a particle velocity transducer, has been hampered by the absence of reliable transducers for the acoustic particle velocity; see, e.g., the historical overview in Ref. 1. The situation is different in underwater acoustics since "in water, direct measurement [of the acoustic particle velocity] is simple,", and several $p-u$ measurement systems have recently been developed; see, e.g., Refs. 4,5. This paper is concerned with air acoustics. Some years ago a micromachined transducer called the "Microflown" became available for measurement of the acoustic particle velocity in air, ${ }^{6}$ and an intensity probe based on this device in combination with a small pressure microphone is now in commercial production. ${ }^{7,8}$ Yet another method based on determining the sound pressure from an approximation to the divergence of the particle velocity (the " $u-u$ " method, which involves six velocity transducers $)^{9}$ has, to the authors' knowledge, never been used in air.

\footnotetext{
${ }^{\mathrm{a})}$ Portions of this work were presented in "A comparison of $p-p$ and $p-u$ sound intensity measurement systems," Proceedings of Eleventh International Congress on Sound and Vibration, St. Petersburg, Russia, July 2004.

${ }^{b)}$ Author to whom correspondence should be addressed; electronic mail: fja@oersted.dtu.dk

${ }^{c)}$ Electronic mail: debree@microflown.com
}

The purpose of this paper is to compare, discuss, and examine the main limitations of the $p-u$ and the $p-p$ measurement principles in sound power determination under very difficult measurement conditions.

\section{THE $p$ - $p$ MEASUREMENT PRINCIPLE}

The $p-p$ measurement principle employs two pressure microphones. The particle velocity component in the direction of the axis of the probe is obtained by a finite-difference approximation to the pressure gradient in Euler's equation of motion, and the sound pressure is simply the average of the two pressure signals. The most important limitations of this measurement technique are caused by the finite difference approximation, scattering and diffraction, and instrumentation phase mismatch.

The accuracy of the finite-difference approximation and the effect of scattering and diffraction obviously depend on the geometry of the microphone arrangement. Several configurations are possible, but in the early 1980s it was shown experimentally that the face-to-face configuration with a solid "spacer" between the two microphones is particularly favorable. ${ }^{10}$ Much later it was discovered that the effect of scattering and diffraction not only tends to counterbalance the finite-difference error but in fact for a certain length of the spacer almost perfectly cancels it under virtually any sound field condition encountered in practice. ${ }^{11}$ A practical consequence is that the upper frequency limit of a sound intensity probe based on two $1 / 2$ in. microphones separated by a $12 \mathrm{~mm}$ spacer in the face-to-face arrangement is about $10 \mathrm{kHz}$, which is about an octave higher than the frequency limit determined by the finite-difference approximation. ${ }^{11}$ The combination of $1 / 2 \mathrm{in}$. microphones and a $12 \mathrm{~mm}$ 
spacer is now regarded as optimal, and longer spacers are only used when the focus is exclusively on low frequencies. $^{12}$

Unless the measurement is compensated for phase mismatch the microphones for measurement of sound intensity with the $p$ - $p$ method have to be phase matched extremely well, and state-of-the-art sound intensity microphones are matched to a maximum phase response difference of $0.05^{\circ}$ below $250 \mathrm{~Hz}$ and a phase difference proportional to the frequency above $250 \mathrm{~Hz}$ (say, $0.2^{\circ}$ at $1 \mathrm{kHz}$ ). ${ }^{13}$ The proportionality to the frequency is a consequence of the fact that phase mismatch in this frequency range is caused by differences between the resonance frequencies and the damping of the two microphones. ${ }^{14}$

It can be shown that a small phase mismatch error $\varphi_{\text {pe }}$ gives rise to a bias error that can be approximated by ${ }^{15,16}$

$$
\hat{I}_{r} \simeq I_{r}-\frac{\varphi_{p e}}{k \Delta r} \frac{p_{\mathrm{rms}}^{2}}{\rho c}=I_{r}\left(1-\frac{\varphi_{p e}}{k \Delta r} \frac{p_{\mathrm{rms}}^{2} / \rho c}{I_{r}}\right),
$$

where $I_{r}$ is the "true" intensity (unaffected by phase mismatch), $\hat{I}_{r}$ is the biased estimate, $p_{\text {rms }}$ is the rms value of the sound pressure, $k$ is the wave number, $\Delta r$ is the microphone separation distance, $\rho$ is the density of air, and $c$ is the speed of sound. This expression shows that the effect of a given phase error is inversely proportional to the frequency and the microphone separation distance and is proportional to the ratio of the mean square sound pressure to the sound intensity. If this ratio is large then even the small phase errors mentioned earlier will give rise to significant bias errors. Because of phase mismatch it will rarely be possible to make reliable measurements below, say, $80 \mathrm{~Hz}$, except under very favorable semi free-field conditions unless a longer spacer than the usual $12 \mathrm{~mm}$ spacer is used. ${ }^{17}$

The ratio of the phase error to the product of the frequency and the microphone separation distance can be measured (usually in the form of the so-called "pressure-residual intensity index"1) by exposing the two pressure microphones to the same pressure in a small coupler. ${ }^{1-3}$ Modern sound intensity analyzers automatically determine the ratio of the mean square pressure to the intensity during the intensity measurements. ${ }^{18}$ Thus one has a clear indication of whether the bias error is serious or not.

The global version of Eq. (1) is found by integrating the normal component over a surface that encloses a source. The result is ${ }^{16,19}$

$$
\hat{P}_{a}=\int_{S} \hat{\mathbf{I}} \cdot d \mathbf{S} \simeq P_{a}\left(1-\frac{\varphi_{p e}}{k \Delta r} \frac{\int_{S}\left(p_{\mathrm{rms}}^{2} / \rho c\right) d S}{\int_{S} \mathbf{I} \cdot d \mathbf{S}}\right),
$$

where $P_{a}$ is the "true" sound power of the source within the surface and $\hat{P}_{a}$ is the biased estimate. The ratio of the surface integral of the mean square pressure to the surface integral of the intensity (in decibels) is known as the pressure-intensity index of the measurement.

Calibration of $p-p$ sound intensity measurement systems involves calibrating the two pressure microphones with a pistonphone in the usual manner and determining the pressure-residual intensity index in a small coupler driven by a wide-band signal as mentioned earlier. ${ }^{1}$

\section{THE $p-u$ MEASUREMENT PRINCIPLE}

\section{A. General considerations}

A $p-u$ sound intensity measurement system combines two fundamentally different transducers. The sound intensity is simply the time average of the instantaneous product of the pressure and particle velocity signal, ${ }^{1}$

$$
I_{r}=\left\langle p u_{r}\right\rangle_{t}=\frac{1}{2} \operatorname{Re}\left\{p u_{r}^{*}\right\},
$$

where \langle\rangle$_{t}$ indicates time averaging, and the latter expression is based on the complex representation of harmonic variables. However, irrespective of the measurement principle used in measuring the particle velocity there is one fundamental problem: the pressure and the particle velocity transducer will invariably have different phase responses. ${ }^{1}$ One must compensate for this " $p-u$ phase mismatch," otherwise the result may well be meaningless. In fact even a small residual $p-u$ mismatch error can have serious consequences under certain conditions. This can be seen by introducing such a small phase error, $\varphi_{u e}$, in Eq. (3). The result is ${ }^{20}$

$$
\begin{aligned}
\hat{I}_{r}=\frac{1}{2} \operatorname{Re}\left\{p \hat{u}_{r}^{*}\right\}= & \frac{1}{2} \operatorname{Re}\left\{p u_{r}^{*} e^{\left.-j \varphi_{u e}\right\}}\right. \\
= & \operatorname{Re}\left\{\left(I_{r}+j J_{r}\right)\left(\cos \varphi_{u e}-j \sin \varphi_{u e}\right)\right\} \simeq I_{r} \\
& +\varphi_{u e} J_{r},
\end{aligned}
$$

where

$$
\hat{u}_{r}=u_{r} e^{j \varphi_{u e}}
$$

is the particle velocity estimate, and

$$
J_{r}=\frac{1}{2} \operatorname{Im}\left\{p u_{r}^{*}\right\}=\operatorname{Re}\left\{I_{r}+j J_{r}\right\}
$$

is the reactive intensity. ${ }^{21,22}$ Whereas the (active) intensity describes the net flow of sound energy the reactive intensity describes the nonpropagating part of the energy, which is merely flowing back and forth, corresponding to the instantaneous particle velocity being in quadrature with the sound pressure. Many sources have strongly reactive near fields at low frequencies where they mainly generate evanescent waves. Near such a source the air is essentially moving back and forth as if it were incompressible. ${ }^{1}$ Equation (4) demonstrates that even a small uncompensated $p-u$ phase mismatch error will give rise to a significant bias error when $J_{r} \gg I_{r}$. On the other hand it also shows that substantial $p$ - $u$ phase errors can be tolerated if $J_{r} \ll I_{r}$. For example, even phase mismatch of $35^{\circ}$ gives a bias error of less than $1 \mathrm{~dB}$ under such conditions. In other words, the phase calibration is critical when measurements are carried out under near field conditions, but not at all critical if the measurements are carried out in the far field. The "reactivity" (the ratio of the reactive to the active intensity) indicates whether this source of error is of concern or not.

The global version of Eq. (4) is found by integrating over a surface that encloses a source, ${ }^{20}$ 


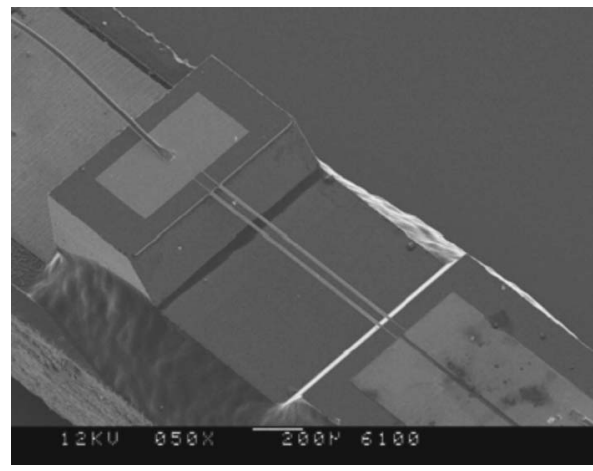

FIG. 1. The two heated wires of the Microflown particle velocity transducer.

$$
\begin{aligned}
\hat{P}_{\mathrm{a}}= & \int_{S} \operatorname{Re}\left\{(\mathbf{I}+j \mathbf{J})\left(\cos \varphi_{u e}-j \sin \varphi_{u e}\right)\right\} \cdot d \mathbf{S} \simeq P_{a} \\
& +\varphi_{u e} \int_{S} \mathbf{J} \cdot d \mathbf{S} \\
= & P_{a}\left(1+\varphi_{u e} \frac{\int_{S} \mathbf{J} \cdot d \mathbf{S}}{\int_{S} \mathbf{I} \cdot d \mathbf{S}}\right),
\end{aligned}
$$

and this shows that uncompensated $p-u$ phase mismatch is a potential source of error when the reactivity (which in the global case is the ratio of the surface integral of the reactive intensity to the surface integral of the active intensity) is large. This will typically occur at low frequencies when the measurement surface is close to the source. Thus the reactivity is an important field indicator for $p-u$ probes. By contrast the pressure-intensity index is not relevant for $p-u$ probes, as also pointed out by Fahy. ${ }^{1}$

Calibration of $p-u$ sound intensity measurement systems involves exposing the probe to a sound field with a known relation between the pressure and the particle velocity, for example a plane propagating wave or a simple spherical wave. ${ }^{1}$ Fahy is rather pessimistic about such calibration techniques, though; he says, "At best this is a check, and not an accurate means of calibration."

\section{B. The Microflown intensity probe}

The MF particle velocity transducer consists of two short, thin, closely spaced wires of silicon nitride coated with platinum and heated by a dc current to about $300{ }^{\circ} \mathrm{C} ;{ }^{6-8}$ see Fig. 1. The resistance of the wires depends on the temperature. An acoustic particle velocity signal in the perpendicular direction changes the temperature distribution instantaneously, because one of the wires will be cooled more than the other by the airflow, and this difference in resistance is measured with a bridge circuit that provides a signal proportional to the particle velocity. At low frequencies the sensitivity of this device increases $6 \mathrm{~dB}$ per octave. Between 100 and $1 \mathrm{kHz}$ the frequency response is relatively flat. Between 1 and $10 \mathrm{kHz}$ there is a rolloff of $6 \mathrm{~dB}$ per octave caused by diffusion effect related with the distance between the two wires, and above $10 \mathrm{kHz}$ the sensitivity decreases an additional $6 \mathrm{~dB}$ per octave because of the thermal heat capacity of the wires. The particle velocity transducer is combined with a small electret condenser microphone in the $1 / 2$ in. sound intensity probe shown in Fig. 2. The velocity trans-

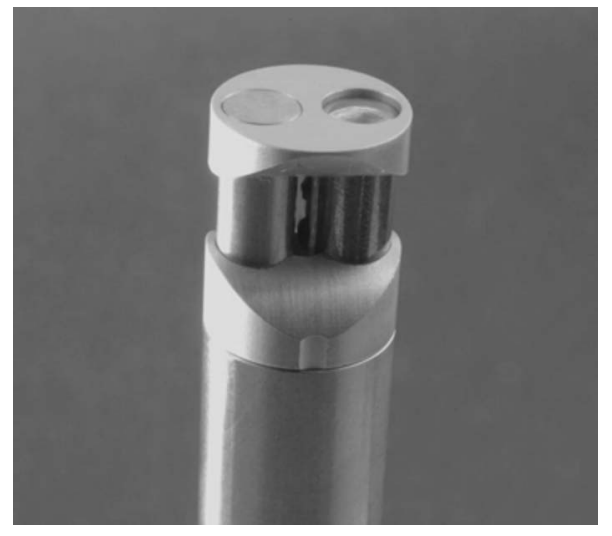

FIG. 2. A Microflown $1 / 2$ in. sound intensity probe. The particle velocity sensor can be seen on the solid cylinder to the right; the electret microphone is mounted inside the other cylinder.

ducer is mounted on a small, solid cylinder, and the condenser microphone is mounted inside another, hollow cylinder. The geometry of this arrangement increases the velocity and thus the sensitivity of the velocity transducer.

\section{DISCUSSION}

Inspection of Eqs. (2) and (7) shows that $p-p$ and $p-u$ sound intensity measurement systems are affected differently by extraneous noise. Sources outside the measurement surface do not contribute to the surface integral of the "true" intensity [in the the denominator of the second term on the right-hand side of Eq. (2)], but they increase the surface integral of the mean square pressure (the numerator of the second term), from which it follows that even a very small phase error imposes restrictions on the amount of extraneous noise that can be tolerated in sound power measurements with a $p-p$ sound intensity measurement system. (This conclusion was anticipated by Pascal as early as in $1981 .^{23}$ ) By contrast, sources outside the measurement surface do not in general increase the reactivity [the second term on the righthand side of Eq. (7)], and thus they do not in general increase the error due to $p-u$ phase mismatch.

High values of the pressure-intensity index can occur in the entire frequency range; ${ }^{11}$ therefore $p$ - $p$ phase mismatch can be of concern also at high frequencies. On the other hand very reactive sound fields are unlikely to occur except at low frequencies; ${ }^{16}$ therefore $p-u$ phase mismatch will generally be a problem only at low frequencies, and it will usually improve the situation to move away from the source.

The simple expedient of reversing a $p$ - $p$ probe makes it possible to eliminate the influence of $p-p$ phase mismatch, because the intensity changes sign but the error does not. ${ }^{24,25}$ Unfortunately, most $p$ - $p$ intensity probes are not symmetrical and therefore not suitable for real measurements with the probe reversed. By contrast the MF probe can easily be reversed. However, reversing a $p-u$ probe simply changes the sign of the result, including the bias error, as easily seen from Eq. (4). ${ }^{26}$ In other words, there is no simple way of getting rid of the phase error of a $p-u$ probe; it must be calibrated with sufficient accuracy. 


\section{EXPERIMENTAL RESULTS}

\section{A. Free-field calibration}

To validate the foregoing considerations and examine the performance of the MF sound intensity probe some experiments have been carried out. Initially the device was tested in the small anechoic room at DTU at a position $2 \mathrm{~m}$ from a small loudspeaker. A Brüel \& Kjær (BK) "pulse" analyzer of type 3560 in one-third octave mode was used in all measurements. First the directional response of the particle velocity sensor was measured in the vertical and in the horizontal plane, and found to be in reasonable agreement with the expected cosine behavior in the entire frequency range. Next, the pressure response was measured; it was found to be in fair agreement with the response of a 1/2 in. free-field microphone of type BK 4191 up to $6.3 \mathrm{kHz}$. And finally the frequency response between the pressure and the velocity signal of the device was determined. This response, which took values over an interval of almost $30 \mathrm{~dB}$ and $140^{\circ}$ in the frequency range from $50 \mathrm{~Hz}$ to $10 \mathrm{kHz}$, served as a correction factor to the cross spectrum in subsequent intensity measurements.

In the first place it was assumed that the probe had been exposed to a plane propagating wave in the velocity calibration measurement. However, as soon as the velocity calibration was applied to sound power measurements it became apparent that $2 \mathrm{~m}$ distance from the source is not enough to ensure plane wave conditions at low frequencies. Thus the measurement was repeated in the large anechoic room at DTU at positions 2, 3, and $4 \mathrm{~m}$ from the loudspeaker. This room is very good at frequencies down to $50 \mathrm{~Hz} .^{27}$ The velocity calibration was corrected for the "near field effect," that is, the factor in parentheses in the expression for the relation between the particle velocity and the pressure generated by a monopole,

$$
u_{r}(r)=\frac{p(r)}{\rho c}\left(1+\frac{1}{j k r}\right) \text {. }
$$

Figure 3 shows the amplitude and phase corrections measured at 2 and $4 \mathrm{~m}$ distance, with and without the near field effect taken into account. (The corrections measured at $3 \mathrm{~m}$ distance are similar.) Below $200 \mathrm{~Hz}$ it is clearly necessary to correct for the near field effect even at a distance of $4 \mathrm{~m}$. The fact that the corrected calibration curves differ from each other below $100 \mathrm{~Hz}$ simply demonstrates that a loudspeaker is not a monopole.

In what follows the active and the reactive intensity has been estimated in one-third octave bands using

$$
\begin{aligned}
& \hat{I}_{r}=\operatorname{Re}\left\{\frac{S_{\hat{p} \hat{u}} H_{p u}}{H_{\hat{p} \hat{u}}\left|H_{p \hat{p}}\right|^{2}}\right\}, \\
& \hat{J}_{r}=-\operatorname{Im}\left\{\frac{S_{\hat{p} \hat{u}} H_{p u}}{H_{\hat{p} \hat{u}}\left|H_{p \hat{p}}\right|^{2}}\right\},
\end{aligned}
$$

where $S_{\hat{p} \hat{u}}$ is the cross spectrum between the two signals from the MF probe, $H_{\hat{p} \hat{u}}$ is the ratio of the velocity to the pressure from the calibration measurement, $H_{p u}$ is the corresponding theoretical value of the ratio of the velocity to the pressure
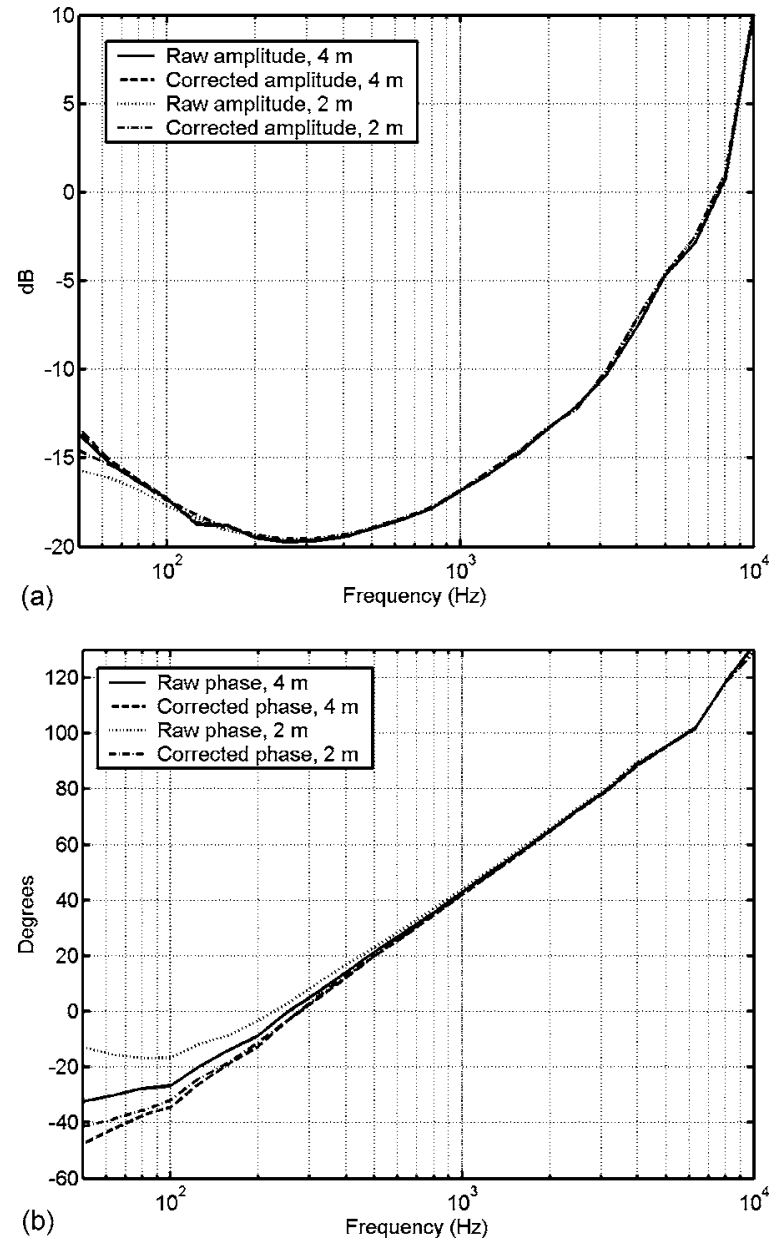

FIG. 3. Amplitude (a) and phase (b) calibration of the velocity signal relative to the pressure signal, measured at 2 and $4 \mathrm{~m}$ distance in an anechoic room, with and without correction for the finite distance to the loudspeaker.

[from Eq. (8)], and the pressure correction, $H_{p \hat{p}}$, takes account of fact that the pressure sensitivity of the device is not completely flat. The pressure correction is shown in Fig. 4.

\section{B. Sound power measurements}

The next experiments took place in a large hall where the sound power of a "sound source" of type BK 4205 was

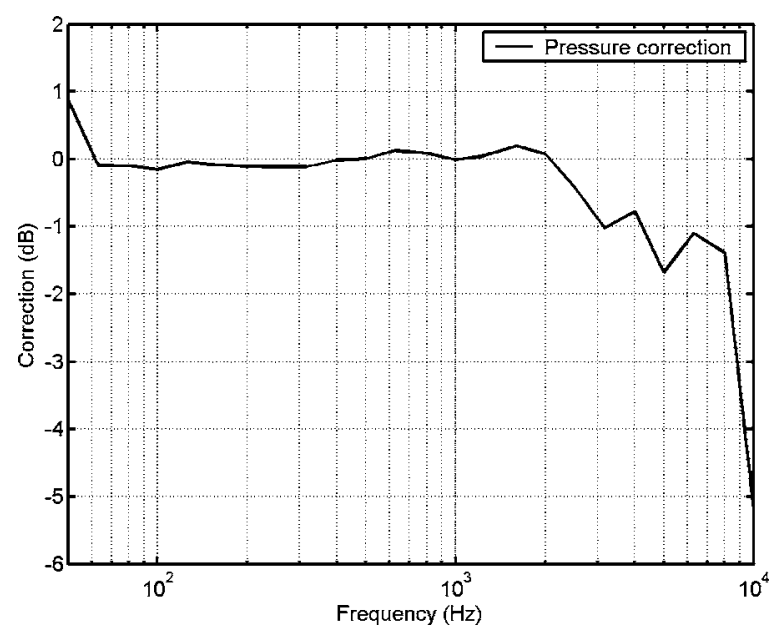

FIG. 4. Pressure correction of the MF probe. 


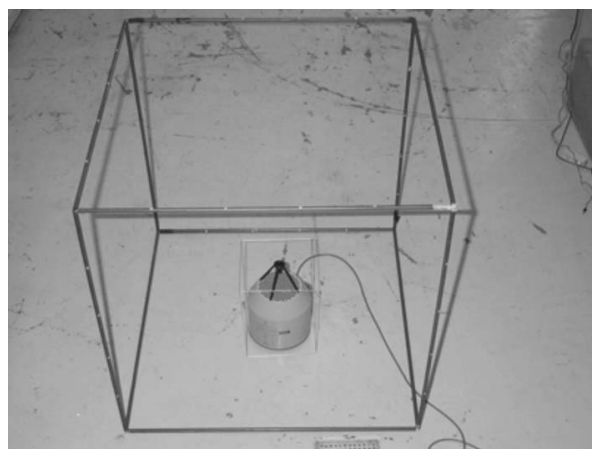

FIG. 5. Large and small measurement surface and the BK 4205 sound source.

measured by scanning over a surface enclosing the source with two different intensity probes, the MF probe and a $p-p$ probe of type BK 3599 with microphones of type BK 4181. The two channels of the BK $p-p$ sound intensity measurement system were much better matched than required of "class 1" systems by the IEC standard; ${ }^{2}$ see Ref. 28. The reactive intensity was also measured. Two different measurement surfaces were used, one with an area of $5 \mathrm{~m}^{2}$, and a very small one with an area of about $0.4 \mathrm{~m}^{2}$ (see Fig. 5), and each measurement was repeated using a different scanning pattern. The repeatability was found to be very good in all cases.

Figure 6 shows the results of the four sound power measurements. The results determined with the MF probe have been processed using the presumably most accurate phase and amplitude calibration function, the one determined at 4 $m$ distance and corrected for the near field effect. As can be seen all four measurements are in good agreement up to 6.3 $\mathrm{kHz}$. At 8 and $10 \mathrm{kHz}$ the MF probe overestimates slightly, perhaps because the pressure response of the device depends on the direction of incidence whereas the pressure calibration has been determined for axial incidence, or perhaps because the geometry of the arrangement affects the particle velocity. No influence of the measurement surface can be seen, in spite of the fact that the sound field on the small surface very

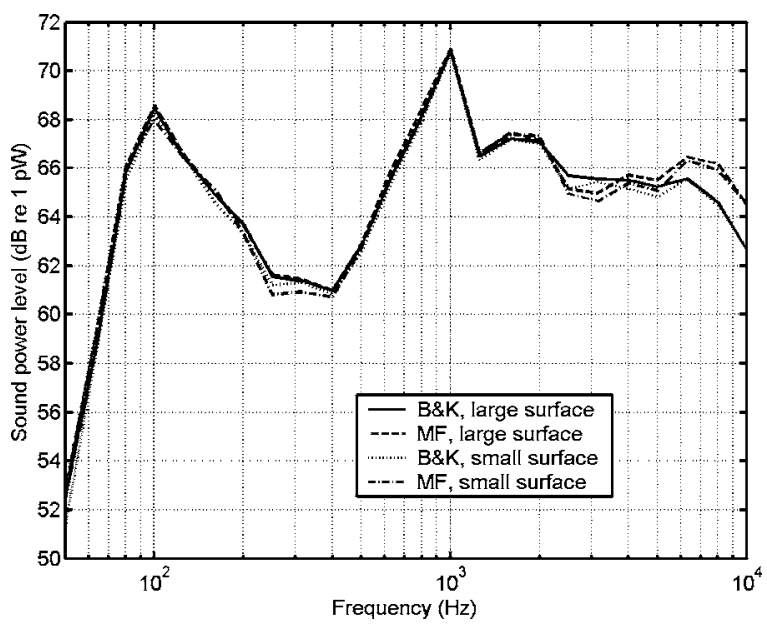

FIG. 6. Sound power of the BK 4205 sound source, measured with a BK intensity probe and with a MF intensity probe on two different measurement surfaces.

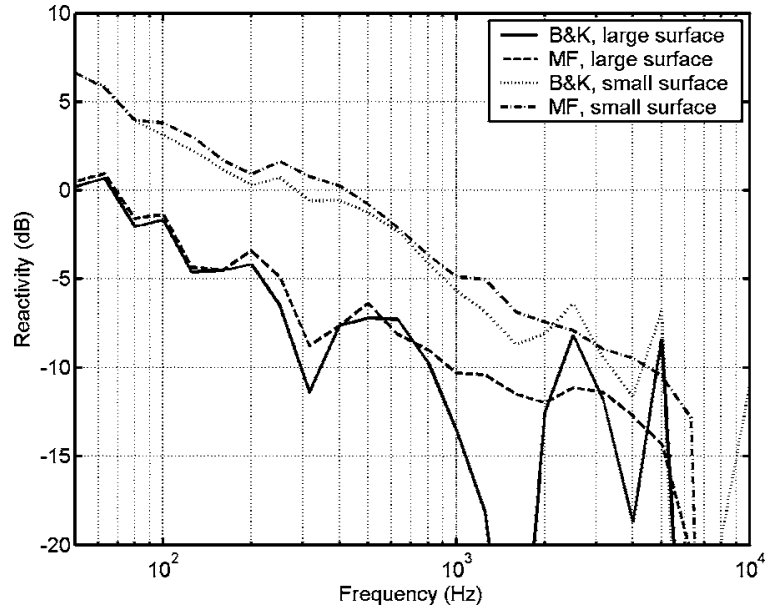

FIG. 7. Ratio of reactive to active intensity on two surfaces enclosing the BK 4205 sound source.

close to the source is strongly reactive at low frequencies, as demonstrated by Fig. 7. The fluctuations in the measured reactivity at high frequencies on the large measurement surface are of no concern. In this frequency range the reactive intensity decays rapidly with the distance to the source, and thus it takes very small values on the large measurement surface. ${ }^{16,26}$ Besides, measuring reactive intensity in a predominantly active sound field with a $p$ - $p$ measurement system requires extremely accurate amplitude calibration. ${ }^{29}$

That the phase calibration of the $p-u$ probe can be critically important is demonstrated by Fig. 8, which shows the error of the sound power measurements with the MF probe on the two measurement surfaces with the velocity calibration measured at 2 and $4 \mathrm{~m}$ distance, without and with correction for the finite distance to the loudspeaker in the calibration measurement. The reference is the measurement with the BK probe on the large surface. It is apparent that the reactive sound field on the small measurement surface amplifies the influence of $p-u$ phase mismatch, as predicted by Eq. (7).

The BK 4205 sound source is an enclosed loudspeaker,

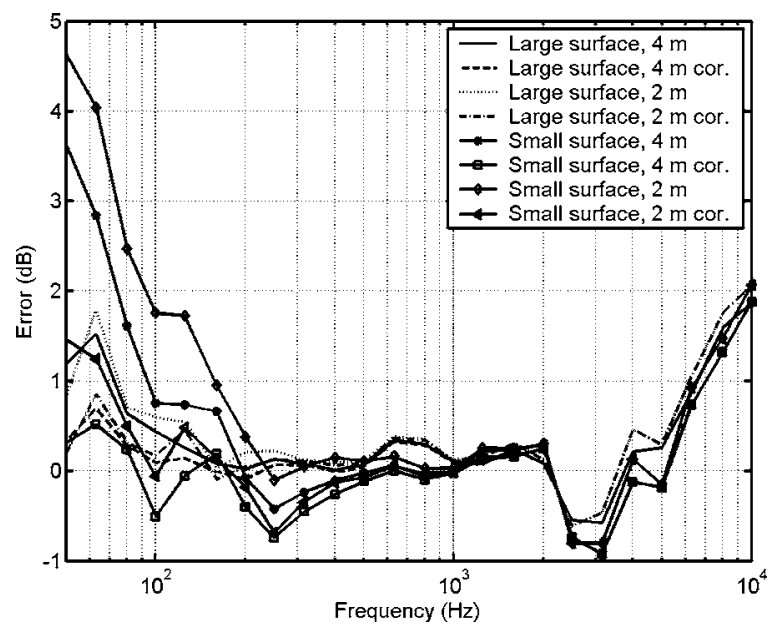

FIG. 8. Estimation error of the MF probe using different phase calibrations. A measurement with a BK intensity probe on the large measurement surface is used as the reference. 


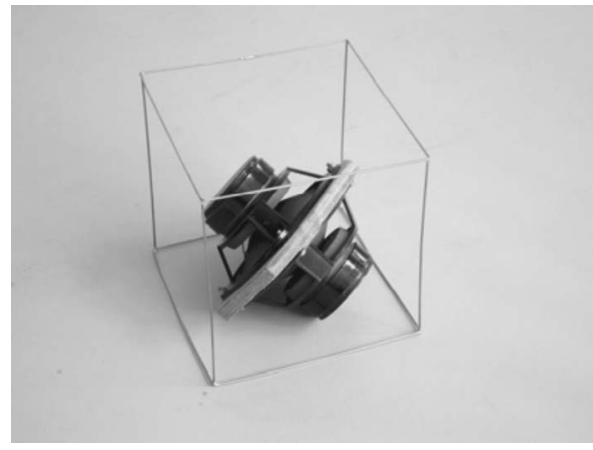

FIG. 9. Dipole source inside a very small measurement surface.

which is a well-behaved source. To examine the performance of the MF probe under more severe conditions the sound power of a dipole has been determined using an extremely small measurement surface with an area of $0.2 \mathrm{~m}^{2}$. The dipole was constructed by mounting two loudspeaker units against each; see Fig. 9. Placed on concrete floor this source generated an exceptionally reactive sound field on the small measurement surface below $500 \mathrm{~Hz}$, as shown in Fig. 10. In this case the BK probe produced negative intensity values at 50 and $63 \mathrm{~Hz}$; hence the missing part of the corresponding curve. However, the "true" average intensity on the small surface can be calculated from the average intensity on the large surface. The MF data on the small surface were affected by residual $p-u$ phase mismatch, as will become apparent in what follows.

Figure 11, which corresponds to Fig. 6, shows the results of sound power measurements with the two devices on the two measurement surfaces. Again, missing parts of the curves indicate meaningless negative sound power estimates. The two probes are in good agreement on the large measurement surface except for the overestimation of the MF probe at 8 and $10 \mathrm{kHz}$, as before. It can also be seen that the BK probe performs reasonably well except at 50 and $63 \mathrm{~Hz}$ on the small surface, considering the difficulties in measuring on such a small surface with the somewhat bulky probe. However, on the small surface the MF probe obviously has problems at low frequencies.

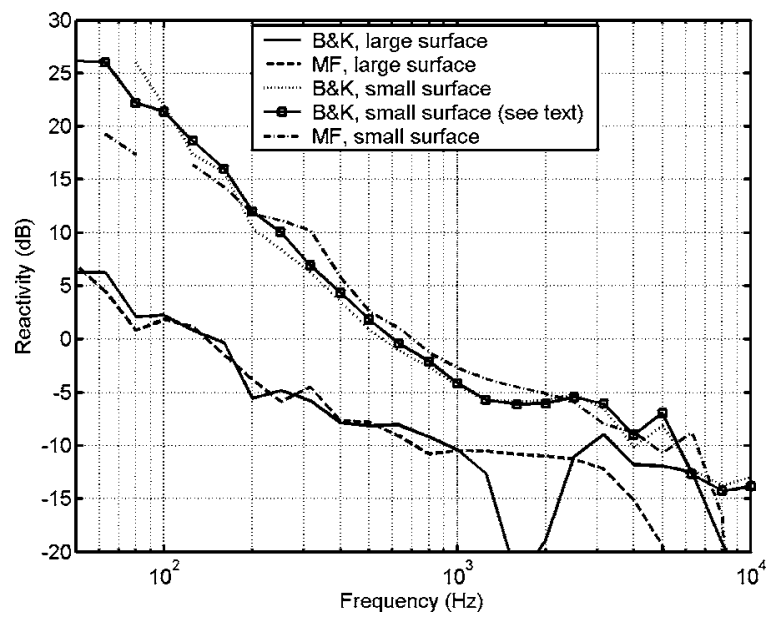

FIG. 10. Ratio of reactive to active intensity on two surfaces enclosing the dipole.

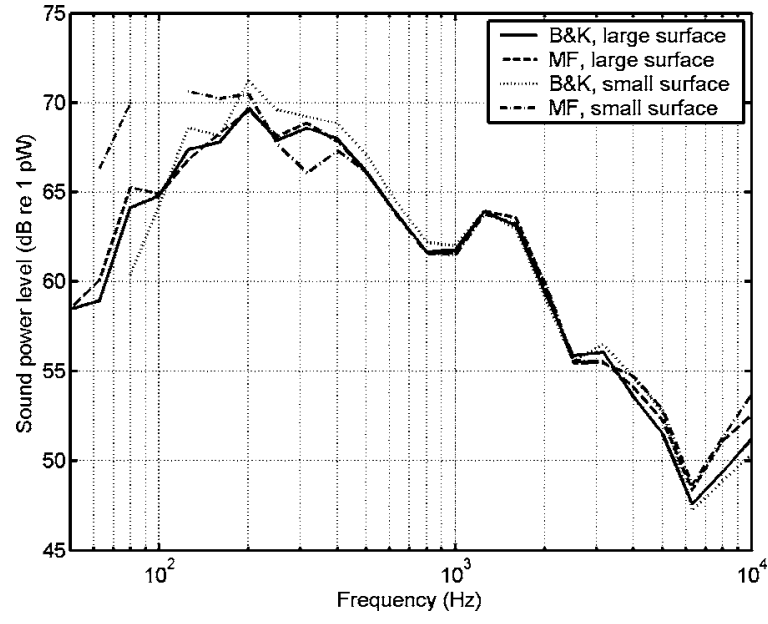

FIG. 11. Sound power of the dipole, measured with a BK intensity probe and with a MF intensity probe on two different measurement surfaces.

Figure 12, which corresponds to Fig. 8, shows the error of the MF results determined using the various calibrations. As can be seen, enormous errors occur when the small measurement surface is used, confirming once again that the phase calibration of the device is critically important when it is used in strongly reactive sound fields.

Finally it should be mentioned that the influence of strong background noise from sources outside the measurement surface has been examined. However, no significant difference between the performance of the $p-p$ and the $p-u$ measurement system under such conditions was observed. ${ }^{28}$ As shown in Sec. II such background noise amplifies the error due to $p$ - $p$ phase mismatch, but with the very wellmatched $p-p$ intensity probe used in these measurements this effect could not be detected. The only effect of the noise was increased random errors, in all probability caused by the fact that the approximation to the surface integral in sound power measurement becomes more critical the higher the level of extraneous noise.

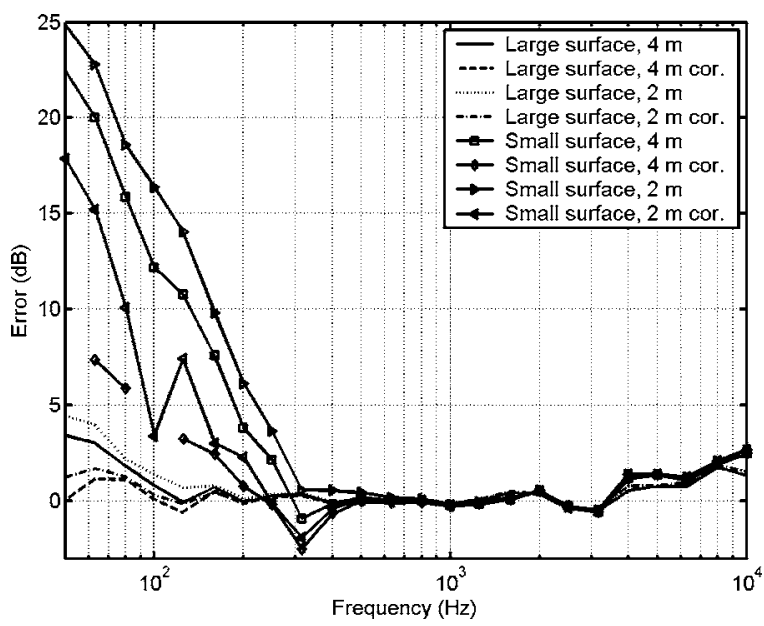

FIG. 12. Estimation error of the MF probe using different phase calibrations. A measurement with a BK intensity probe on the large measurement surface is used as the reference. 


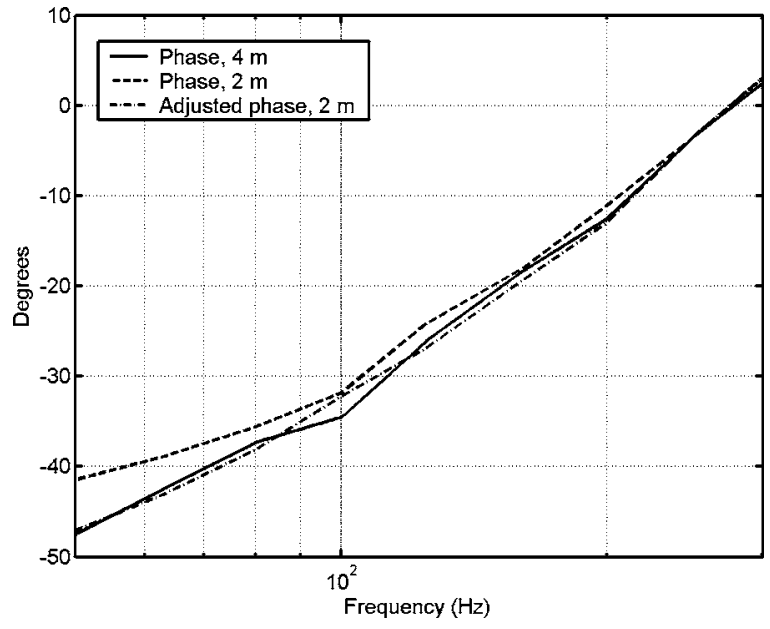

FIG. 13. Original and adjusted phase calibration.

\section{Improved phase calibration}

Not everybody has easy access to a large anechoic room of high quality; therefore it has been examined whether one can improve a free-field calibration made too close to the loudspeaker simply by manual adjustment of the phase calibration until the error that occurs in a very reactive sound field disappears. Figure 13 shows the modified phase correction, and Fig. 14 shows the result of such a procedure in the frequency range from 50 to $200 \mathrm{~Hz}$. The starting point was the phase calibration measured at $2 \mathrm{~m}$ distance and corrected for the phase angle between pressure and particle velocity due to the finite distance on the assumption that the loudspeaker was a perfect monopole. The adjustment must be made in small steps. Below $100 \mathrm{~Hz}$ a phase increment of $0.1^{\circ}$ has drastic consequences for the measurement on the small surface (the intensity estimate can change its sign, for example), confirming again that the phase calibration is critically important under such conditions. Modifying the phase calibration so as to remove the overestimation in the results on the small measurement surface at low frequencies has a very limited effect on the results determined on the large measurement surface, and also, not shown, a very modest influence on the measurement of the sound power of the BK

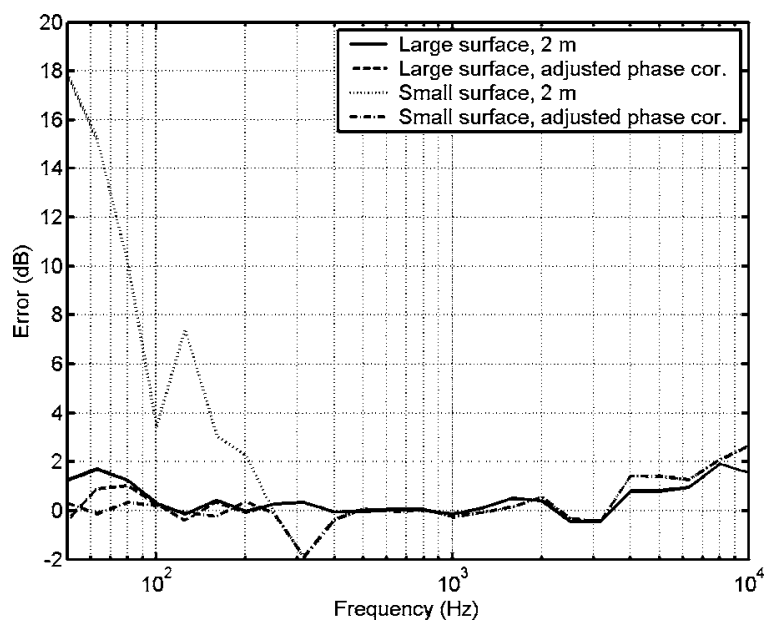

FIG. 14. Estimation error with improved calibration.
4205 using the large surface, but a positive influence on the (fairly small) error in the measurement of the sound power of the BK 4205 made on the small surface (not shown).

Obviously this method cannot take account of errors in the amplitude calibration. However, these errors are quite small, cf. Fig. 3(a), and their effect do not depend on the sound field conditions.

\section{CONCLUSIONS}

The $p-u$ sound intensity measurement principle has been examined theoretically and experimentally, and compared with the established $p-p$ method with particular regard to the influence of phase mismatch on sound power estimation. It is more difficult to calibrate $p$ - $u$ intensity probes than $p$ - $p$ intensity probes, and whereas $p-p$ phase mismatch in conventional $p-p$ measurement systems can be almost eliminated by reversing the intensity probe, probe reversal has no effect on the influence of $p-u$ phase mismatch in measurements with $p-u$ measurement systems. Strongly reactive sound fields exacerbate the influence of $p-u$ phase mismatch, but have no influence on the effect of $p$ - $p$ phase mismatch. Such sound field conditions are reflected in a high value of the reactivity, that is, the ratio of the surface average of the reactive intensity to the surface average of the active intensity. This will rarely be a problem above $500 \mathrm{~Hz}$, and can be avoided simply by moving the measurement surface further away from the source under investigation. By contrast, background noise from sources outside the measurement surface increases the influence of $p-p$ phase mismatch, but has no influence on the effect of $p-u$ phase mismatch. Such background noise is reflected in a high value of the pressureintensity index. This problem can occur in the entire frequency range.

The experimental part of the investigation was carried out with a $p-u$ sound intensity probe produced by Microflown. The results show that it is possible to measure sound power reliably with the Microflown intensity probe from $50 \mathrm{~Hz}$ to $6.3 \mathrm{kHz}$ if strongly reactive near fields, which tend to make the phase calibration very critical, are avoided. The measurements have also shown that it is possible to expand the range of measurement to near field conditions if the phase can be calibrated with sufficient accuracy below $500 \mathrm{~Hz}$; at higher frequencies the phase calibration is less critical. The calibration can be carried out in a large anechoic room, and the extreme sensitivity to $p-u$ phase mismatch in very reactive sound fields can even be turned to advantage by adjustment of the phase correction until the error disappears.

\section{ACKNOWLEDGMENT}

The authors would like to thank Johan Gramtorp for the idea of adjusting the phase angle of the velocity correction until the error in a strongly reactive sound field disappears.

${ }^{1}$ F. J. Fahy, Sound Intensity 2nd ed. (E \& FN Spon, London, 1995).

${ }^{2}$ IEC Publication 61043, "Instruments for the measurement of sound intensity-Measurements with pairs of pressure sensing microphones," 1993.

${ }^{3}$ American National Standard ANSI S1.9-1996, "Instruments for the mea- 
surement of sound intensity," 1996.

${ }^{4}$ T. B. Gabrielson, D. L. Gardner, and S. T. Garret, "A simple neutrally buoyant sensor for direct measurement of particle velocity and intensity in water," J. Acoust. Soc. Am. 97, 2227-2237 (1995).

${ }^{5}$ K. Kim, T. Gabrielson, and G. C. Lauchle, "Development of an accelerometer-based underwater acoustic intensity sensor," J. Acoust. Soc. Am. 116, 3384-3392 (2004).

${ }^{6}$ H.-E. de Bree, P. Leussink, T. Korthorst, H. Jansen, T. Lammerink, and M. Elwensoek, "The Microflown: A novel device measuring acoustical flows," Sens. Actuators, A SNA054/1-3, 552-557 (1996).

${ }^{7}$ H.-E. de Bree, "The Microflown: An acoustic particle velocity sensor," Acoust. Aust. 31, 91-94 (2003).

${ }^{8}$ R. Raangs, W. F. Druyvesteyn, and H.-E. de Bree, "A low-cost intensity probe," J. Audio Eng. Soc. 51, 344-357 (2003).

${ }^{9}$ K. J. Bastyr, G. C. Lauchle, and J. A. McConnell, "Development of a velocity gradient underwater acoustic intensity sensor," J. Acoust. Soc. Am. 106, 3178-3188 (1999).

${ }^{10}$ G. Rasmussen and M. Brock, "Acoustic intensity measurement probe," Proceedings of Recent Developments in Acoustic Intensity Measurement, Senlis, France, 1981, pp. 81-88.

${ }^{11}$ F. Jacobsen, V. Cutanda, and P. M. Juhl, “A numerical and experimental investigation of the performance of sound intensity probes at high frequencies," J. Acoust. Soc. Am. 103, 953-961 (1998).

${ }^{12}$ F. Jacobsen, V. Cutanda, and P. M. Juhl, "A sound intensity probe for measuring from $50 \mathrm{~Hz}$ to $10 \mathrm{kHz}$," Brüel \& Kjær Tech. Rev. 1, 1-8 (1996).

${ }^{13}$ Anonymous, "Product data, sound intensity pair-type 4197," Brüel \& Kjær, Nærum, Denmark.

${ }^{14}$ E. Frederiksen and O. Schultz, "Pressure microphones for intensity measurements with significantly improved phase properties," Brüel \& Kjær Tech. Rev. 4, 11-12 (1986).

${ }^{15}$ T. G. Nielsen, "A field calibrated real time system for intensity measurements," Proceedings of Inter-Noise 88, Avignon, France, 1988, pp. $123-126$.
${ }^{16}$ F. Jacobsen, "Sound field indicators: Useful tools," Noise Control Eng. J. 35, 37-46 (1990)

${ }^{17} \mathrm{~S}$. Gade, "Validity of intensity measurements in partially diffuse sound field," Brüel \& Kjær Tech. Rev. 4, 3-31 (1985).

${ }^{18}$ Anonymous, "Technical documentation, sound intensity software BZ 7205 for 2260 Investigator," Brüel \& Kjær, Nærum, Denmark.

${ }^{19} \mathrm{~F}$. Jacobsen, "A simple and effective correction for phase mismatch in intensity probes," Appl. Acoust. 33, 165-180 (1991).

${ }^{20} \mathrm{~F}$. Jacobsen, "Spatial sampling errors in sound power estimation based upon intensity," J. Sound Vib. 145, 129-149 (1991).

${ }^{21}$ J. A. Mann, III, J. Tichy, and A. J. Romano, "Instantaneous and timeaveraged energy transfer in acoustic fields," J. Acoust. Soc. Am. 82, 17-30 (1987).

${ }^{22}$ F. Jacobsen, "Active and reactive, coherent and incoherent sound fields," J. Sound Vib. 130, 493-507 (1989).

${ }^{23}$ J.-C. Pascal, "Estimation de la puissance acoustique à l'aide d'intensimètres," Proceedings of Recent Developments in Acoustic Intensity Measurement, Senlis, France, 1981, pp. 179-185.

${ }^{24}$ J. Y. Chung, "Cross-spectral method of measuring acoustic intensity without error caused by instrument phase mismatch," J. Acoust. Soc. Am. 64, 1613-1616 (1978).

${ }^{25}$ S. J. Elliott, "Errors in acoustic intensity measurements," J. Sound Vib. 78, 439-445 (1981).

${ }^{26}$ F. Jacobsen and E. S. Olsen, "The influence of microphone vents on the performance of sound intensity probes," Appl. Acoust. 41, 25-45 (1994).

${ }^{27}$ F. Ingerslev, O. J. Pedersen, P. K. Møller, and J. Kristensen, "New rooms for acoustic measurements at the Danish Technical University," Acustica 19, 185-199 (1967/68).

${ }^{28}$ F. Jacobsen and H.-E. de Bree, "Intensity-based sound power determination under adverse sound field conditions: p-p versus p-u probes," Proceedings of Twelfth International Congress on Sound and Vibration, Lisbon, Portugal, 2005.

${ }^{29} \mathrm{~F}$. Jacobsen, "Active and reactive sound intensity in a reverberant sound field," J. Sound Vib. 143, 231-240 (1990). 